Supporting information for

\title{
Tunable Perovskite-Derived Bismuth Halides:
}

\author{
$\mathrm{Cs}_{3} \mathrm{Bi}_{2}\left(\mathrm{Cl}_{1-x} \mathrm{l}_{x}\right)_{9}$
}

Emily E. Morgan,1,2 Lingling Mao, 1,* Samuel M. L. Teicher, 1,2 Guang Wu ${ }^{3}$, and Ram Seshadri, 2,3

${ }^{1}$ Materials Research Laboratory

${ }^{2}$ Materials Department

${ }^{3}$ Department of Chemistry and Biochemistry

University of California, Santa Barbara, California 93106, United States

*Email: linglingmao@ucsb.edu

Table of contents

Section S1. Additional crystallographic details

Section S2. Rietveld analysis of $\mathrm{Cs}_{3} \mathrm{Bi}_{2}\left(\mathrm{Cl}_{1-x} \mathrm{l}_{x}\right)_{9}$ compounds

Section S3. Additional calculation data 
Section S1. Additional crystallographic details

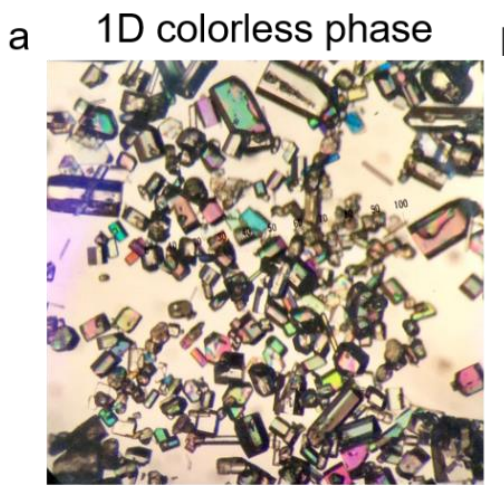

$\overline{0.67 \mathrm{~mm}}$

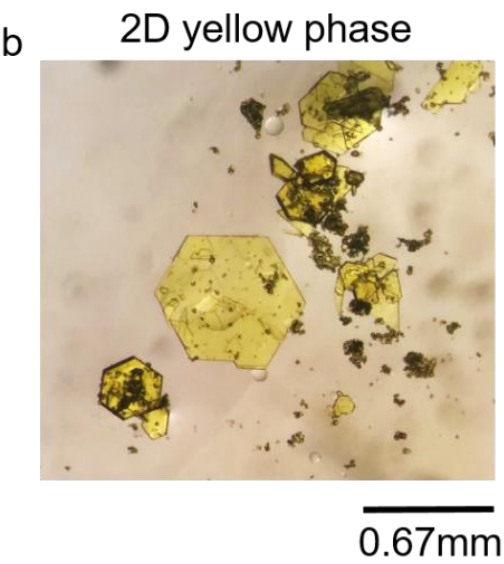

Figure S1. Optical microscopic images of the ${ }_{1} \mathrm{D}$ colorless phase and $2 \mathrm{D}$ yellow phase of $\mathrm{Cs}_{3} \mathrm{Bi}_{2} \mathrm{Cl}_{9}$ and $\mathrm{Cs}_{3} \mathrm{Bi}_{2} \mathrm{Cl}_{8.16} \mathrm{I}_{0.84}$.

Table S1. Atomic coordinates $\left(\times 10^{4}\right)$ and equivalent isotropic displacement parameters $\left(\AA^{2} \times 10^{3}\right)$ for $\mathrm{Cs}_{3} \mathrm{Bi}_{2} \mathrm{Cl}_{9}$ with estimated standard deviations in parentheses.

\begin{tabular}{llllll}
\hline Label & $x$ & $y$ & $z$ & Occupancy & $\mathrm{U}_{\mathrm{eq}}{ }^{*}$ \\
\hline $\mathrm{Bi}(\mathrm{o1})$ & $6833(1)$ & 7500 & $4179(1)$ & 1 & $18(1)$ \\
$\mathrm{Bi}(02)$ & $4988(1)$ & 2500 & $2363(1)$ & 1 & $19(1)$ \\
$\mathrm{Cs}(03)$ & $9112(1)$ & 12500 & $5811(1)$ & 1 & $40(1)$ \\
$\mathrm{Cs}(04)$ & $7518(1)$ & 2500 & $2521(1)$ & 1 & $42(1)$ \\
$\mathrm{Cs}(05)$ & $9135(1)$ & 7500 & $892(1)$ & 1 & $44(1)$ \\
$\mathrm{Cl}(\mathrm{o6})$ & $4200(1)$ & $77(2)$ & $1633(2)$ & 1 & $43(1)$ \\
$\mathrm{Cl}(07)$ & $5885(2)$ & $5032(2)$ & $3321(2)$ & 1 & $53(1)$ \\
$\mathrm{Cl}(\mathrm{o} 8)$ & $5797(2)$ & 2500 & $820(2)$ & 1 & $45(1)$ \\
$\mathrm{Cl}(09)$ & $7511(1)$ & $10043(2)$ & $5037(2)$ & 1 & $41(1)$ \\
$\mathrm{Cl}(\mathrm{oA})$ & $7539(2)$ & 7500 & $2503(2)$ & 1 & $42(1)$ \\
$\mathrm{Cl}(\mathrm{oB})$ & $5873(2)$ & 7500 & $5833(2)$ & 1 & $50(1)$
\end{tabular}

${ }^{\text {*}} U_{\text {eq }}$ is defined as one third of the trace of the orthogonalized $U_{i j}$ tensor. 
Table S2. Atomic coordinates $\left(\times 10^{4}\right)$ and equivalent isotropic displacement parameters $\left(\AA^{2} \times 10^{3}\right)$ for $\mathrm{Cs}_{3} \mathrm{Bi}_{2} \mathrm{Cl}_{8.16} \mathrm{I}_{0.84}$ with estimated standard deviations in parentheses.

\begin{tabular}{llllll}
\hline Label & $x$ & $y$ & $z$ & Occupancy & $U_{\mathrm{eq}}{ }^{*}$ \\
\hline $\mathrm{Bi}$ & 6666.67 & 3333.33 & $8161(1)$ & 1 & $23(1)$ \\
$\mathrm{Cs}(1)$ & 3333.33 & 6666.67 & $6661(2)$ & 1 & $58(1)$ \\
$\mathrm{Cs}(2)$ & $\mathrm{o}$ & $\mathrm{o}$ & $\mathrm{o}$ & 1 & $71(1)$ \\
$\mathrm{Cl}(1)$ & $3327(3)$ & $1664(2)$ & $6626(2)$ & $0.861(5)$ & $64(1)$ \\
$\mathrm{I}(1)$ & $3327(3)$ & $1664(2)$ & $6626(2)$ & $0.139(5)$ & $64(1)$ \\
$\mathrm{Cl}(2)$ & 5000 & 5000 & 0 & 1 & $71(2)$ \\
\hline
\end{tabular}

${ }^{*} U_{\text {eq }}$ is defined as one third of the trace of the orthogonalized $U_{i j}$ tensor.

Table S3. Anisotropic displacement parameters $\left(\AA^{2} \times 10^{3}\right)$ for $\mathrm{Cs}_{3} \mathrm{Bi}_{2} \mathrm{Cl}_{9}$ with estimated standard deviations in parentheses.

\begin{tabular}{lllllll}
\hline Label & $U_{11}$ & $U_{22}$ & $U_{33}$ & $U_{12}$ & $U_{13}$ & $U_{23}$ \\
\hline $\mathrm{Bi}(01)$ & $19(1)$ & $19(1)$ & $15(1)$ & 0 & $0(1)$ & 0 \\
$\mathrm{Bi}(02)$ & $18(1)$ & $21(1)$ & $18(1)$ & 0 & $0(1)$ & 0 \\
$\mathrm{Cs}(03)$ & $45(1)$ & $44(1)$ & $32(1)$ & 0 & $1(1)$ & 0 \\
$\mathrm{Cs}(04)$ & $38(1)$ & $46(1)$ & $43(1)$ & 0 & $8(1)$ & 0 \\
$\mathrm{Cs}(05)$ & $45(1)$ & $40(1)$ & $46(1)$ & 0 & $-1(1)$ & 0 \\
$\mathrm{Cl}(06)$ & $47(1)$ & $39(1)$ & $44(1)$ & $-17(1)$ & $-9(1)$ & $-8(1)$ \\
$\mathrm{Cl}(07)$ & $52(2)$ & $46(1)$ & $60(2)$ & $-21(1)$ & $-13(2)$ & $-15(1)$ \\
$\mathrm{Cl}(08)$ & $46(2)$ & $58(2)$ & $32(2)$ & 0 & $20(2)$ & 0 \\
$\mathrm{Cl}(09)$ & $40(1)$ & $36(1)$ & $46(1)$ & $-11(1)$ & $-7(1)$ & $-12(1)$ \\
$\mathrm{Cl}(\mathrm{oA})$ & $40(2)$ & $57(2)$ & $27(2)$ & 0 & $17(1)$ & 0 \\
$\mathrm{Cl}(\mathrm{oB})$ & $50(2)$ & $66(2)$ & $34(2)$ & 0 & $23(2)$ & 0 \\
\hline
\end{tabular}

The anisotropic displacement factor exponent takes the form: $-2 \pi^{2}\left[h^{2} a^{*} U_{11}+\ldots+2 h k a^{*} b^{*} U_{12}\right]$. 
Table S4. Anisotropic displacement parameters $\left(\AA^{2} \times 10^{3}\right)$ for $\mathrm{Cs}_{3} \mathrm{Bi}_{2} \mathrm{Cl}_{8.16} \mathrm{I}_{0.84}$ with estimated standard deviations in parentheses.

\begin{tabular}{lllllll}
\hline Label & $\mathrm{U}_{11}$ & $\mathrm{U}_{22}$ & $\mathrm{U}_{33}$ & $\mathrm{U}_{12}$ & $\mathrm{U}_{13}$ & $\mathrm{U}_{23}$ \\
\hline $\mathrm{Bi}$ & $25(1)$ & $25(1)$ & $19(1)$ & $13(1)$ & $\mathrm{o}$ & $\mathrm{o}$ \\
$\mathrm{Cs}(1)$ & $64(1)$ & $64(1)$ & $46(1)$ & $32(1)$ & 0 & 0 \\
$\mathrm{Cs}(2)$ & $57(1)$ & $57(1)$ & $99(2)$ & $28(1)$ & 0 & 0 \\
$\mathrm{Cl}(1)$ & $70(2)$ & $69(1)$ & $53(1)$ & $35(1)$ & $-12(1)$ & $-6(1)$ \\
$\mathrm{I}(1)$ & $70(2)$ & $69(1)$ & $53(1)$ & $35(1)$ & $-12(1)$ & $-6(1)$ \\
$\mathrm{Cl}(2)$ & $84(2)$ & $84(2)$ & $69(2)$ & $60(2)$ & $18(1)$ & $-18(1)$ \\
\hline
\end{tabular}

The anisotropic displacement factor exponent takes the form: $-2 \pi^{2}\left[h^{2} a^{*} U_{11}+\ldots+2 h k a^{*} b^{*} U_{12}\right]$.

Table $\mathrm{S}_{5}$. Selected bond lengths $[\AA]$ for $\mathrm{Cs}_{3} \mathrm{Bi}_{2} \mathrm{Cl}_{9}$ with estimated standard deviations in parentheses.

\begin{tabular}{|c|c|}
\hline Label & Distances \\
\hline $\mathrm{Bi}(\mathrm{o1})-\mathrm{Cl}(\mathrm{0} 7) \# 3$ & $2.8180(17)$ \\
\hline $\mathrm{Bi}(\mathrm{o1})-\mathrm{Cl}(\mathrm{o} 7)$ & $2.8181(17)$ \\
\hline $\mathrm{Bi}(01)-\mathrm{Cl}(09)$ & $2.5748(17)$ \\
\hline $\mathrm{Bi}(01)-\mathrm{Cl}(09) \# 3$ & $2.5748(17)$ \\
\hline $\mathrm{Bi}(\mathrm{o1})-\mathrm{Cl}(\mathrm{oA})$ & $2.572(2)$ \\
\hline $\mathrm{Bi}(\mathrm{ol})-\mathrm{Cl}(\mathrm{oB})$ & $2.823(3)$ \\
\hline $\mathrm{Bi}(02)-\mathrm{Cl}(06)$ & $2.5475(16)$ \\
\hline $\mathrm{Bi}(02)-\mathrm{Cl}(06) \# 8$ & $2.5475(16)$ \\
\hline $\mathrm{Bi}(\mathrm{o2})-\mathrm{Cl}(\mathrm{o} 7) \# 8$ & $2.8487(17)$ \\
\hline $\mathrm{Bi}(02)-\mathrm{Cl}(07)$ & $2.8487(17)$ \\
\hline $\mathrm{Bi}(02)-\mathrm{Cl}(\mathrm{o} 8)$ & $2.532(2)$ \\
\hline $\mathrm{Bi}(\mathrm{o} 2)-\mathrm{Cl}(\mathrm{oB}) \# 9$ & $2.870(3)$ \\
\hline
\end{tabular}

Symmetry transformations used to generate equivalent atoms:

(1) $x, y+1, z(2)-x+3 / 2,-y+1, z+1 / 2(3) x,-y+3 / 2, z(4)-x+3 / 2,-y+2, z-1 / 2(5) x-1 / 2, y-1,-z+1 / 2(6)-x+3 / 2,-$ $\mathrm{y}+1, \mathrm{z}-1 / 2(7) \mathrm{x}-1 / 2, \mathrm{y},-\mathrm{z}+1 / 2$ (8) $\mathrm{x},-\mathrm{y}+1 / 2, \mathrm{z}$ (9) $-\mathrm{x}+1,-\mathrm{y}+1,-\mathrm{z}+1$ (10) $-\mathrm{x}+3 / 2, \mathrm{y}+3 / 2, \mathrm{z}+1 / 2$ (11) $\mathrm{x}+1 / 2,-\mathrm{y}+3 / 2,-$ $\mathrm{z}+1 / 2(12) \mathrm{x}+1 / 2, \mathrm{y}+1,-\mathrm{z}+1 / 2(13)-\mathrm{x}+3 / 2, \mathrm{y}+1 / 2, \mathrm{z}+1 / 2(14)-\mathrm{x}+3 / 2,-\mathrm{y}+2, \mathrm{z}+1 / 2(15) \mathrm{x},-\mathrm{y}+5 / 2, \mathrm{z}(16) \mathrm{x}+1 / 2, \mathrm{y},-$ $\mathrm{z}+1 / 2(17) \mathrm{x}+1 / 2,-\mathrm{y}+1 / 2,-\mathrm{z}+1 / 2(18) \mathrm{x}, \mathrm{y}-1, \mathrm{z}(19)-\mathrm{x}+3 / 2, \mathrm{y}-1 / 2, \mathrm{z}-1 / 2(20)-\mathrm{x}+3 / 2, \mathrm{y}+1 / 2, \mathrm{z}-1 / 2$ 
Table S6. Bond lengths $[\AA]$ for $\mathrm{Cs}_{3} \mathrm{Bi}_{2} \mathrm{Cl}_{8.16} \mathrm{I}_{0.84}$ with estimated standard deviations in parentheses.

\begin{tabular}{l|l}
\hline Label & Distances \\
\hline $\operatorname{Bi}(01)-C l(1) \# 1$ & $2.6772(19)$ \\
$\operatorname{Bi}(01)-C l(2) \# 1$ & $2.8428(2)$ \\
\hline
\end{tabular}

Symmetry transformations used to generate equivalent atoms:

(1) $-y+1, x-y, z(2)-x+y+1,-x+1, z(3) y,-x+y+1,-z+1(4) x-y, x,-z+1$ (5) -x+1,-y+1,-z+1 (6) -y,x-y,z (7) x,y+1,z

(8) $x-1, y-1, z(9)-x+y,-x+1, z(10)-x+y,-x, z(11) x, y-1, z(12)-x+1,-y+1,-z+2$ (13) $x+1, y+1, z$

\section{Section S2. Rietveld analysis of $\mathrm{Cs}_{3} \mathrm{Bi}_{2}\left(\mathrm{Cl}_{1-\mathrm{x}} \mathrm{I}_{\mathbf{x}}\right)_{9}$ compounds}

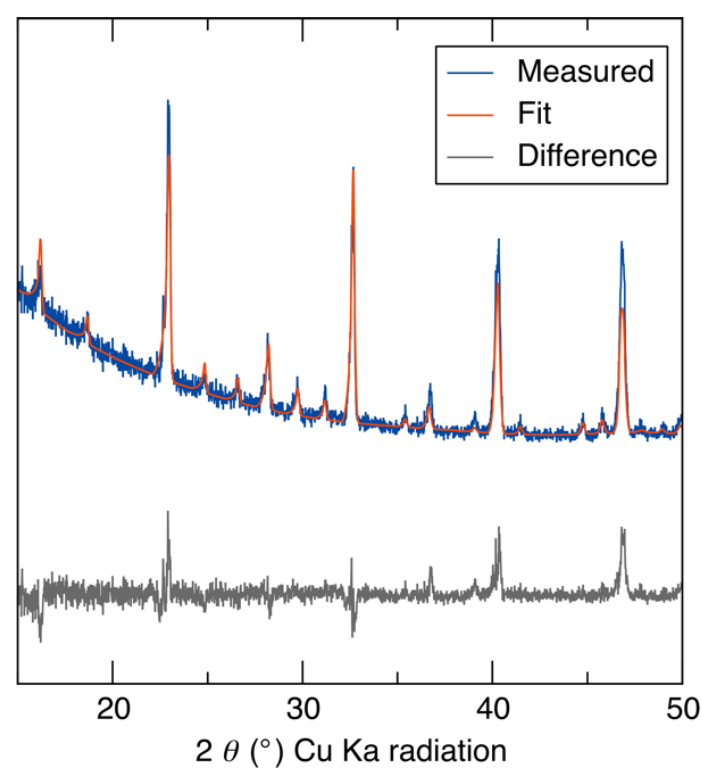

Figure S2. Rietveld refinement for compound with $x=0.095$ showing the PXRD data, the Rietveld fit produced by TOPAS, and the difference between them. 


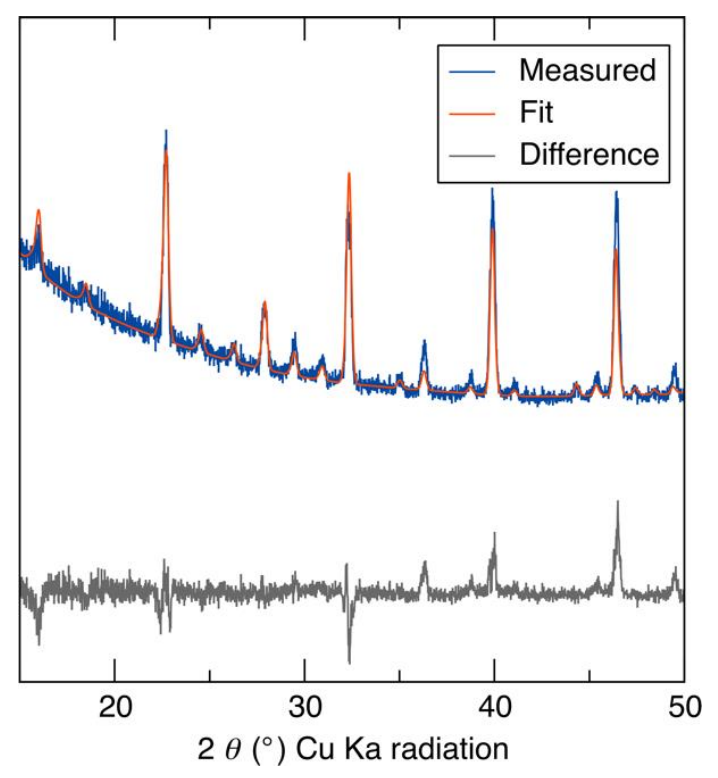

Figure S3. Rietveld refinement for compound with $x=0.16$ showing the PXRD data, the Rietveld fit produced by TOPAS, and the difference between them.

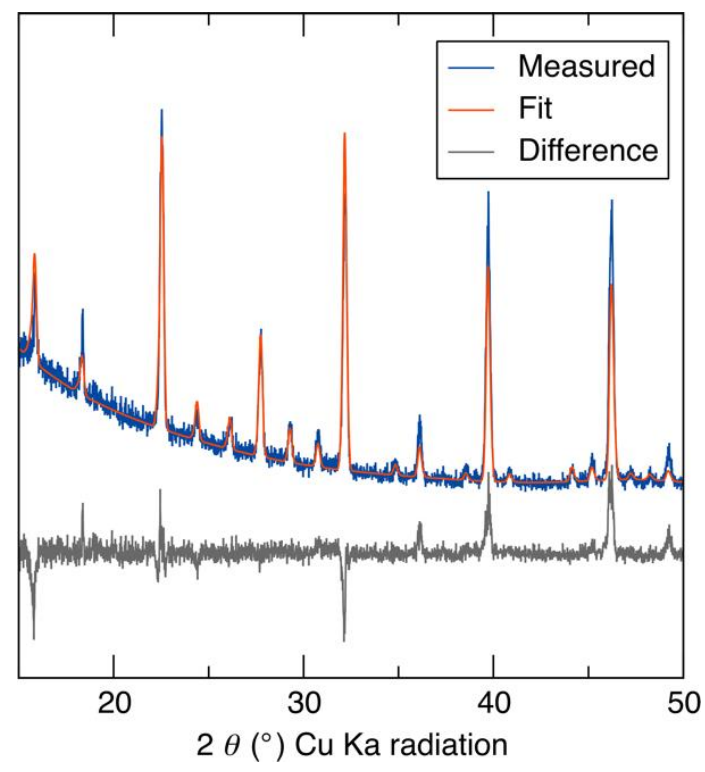

Figure S4. Rietveld refinement for compound with $x=0.18$ showing the PXRD data, the Rietveld fit produced by TOPAS, and the difference between them. 


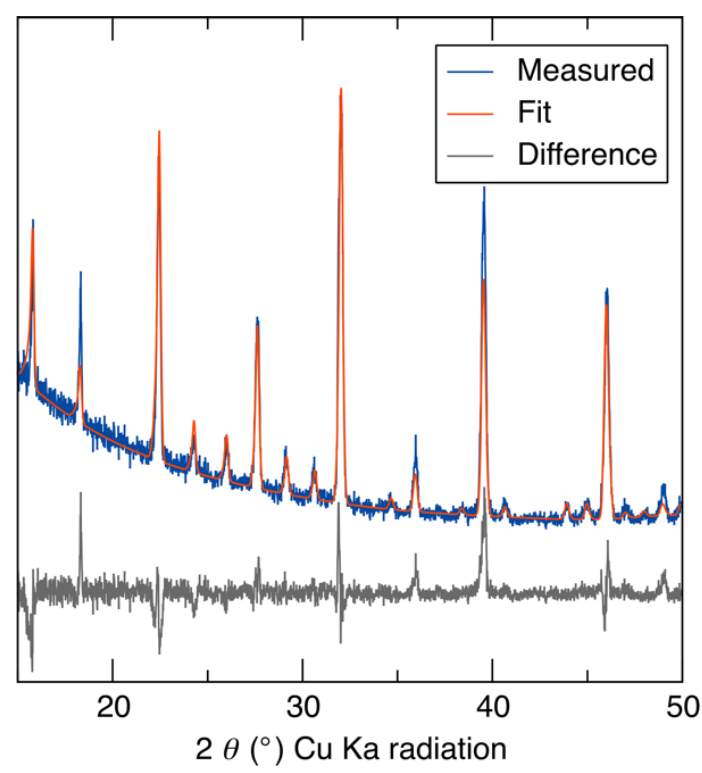

Figure S5. Rietveld refinement for compound with $x=0.23$ showing the PXRD data, the Rietveld fit produced by TOPAS, and the difference between them.

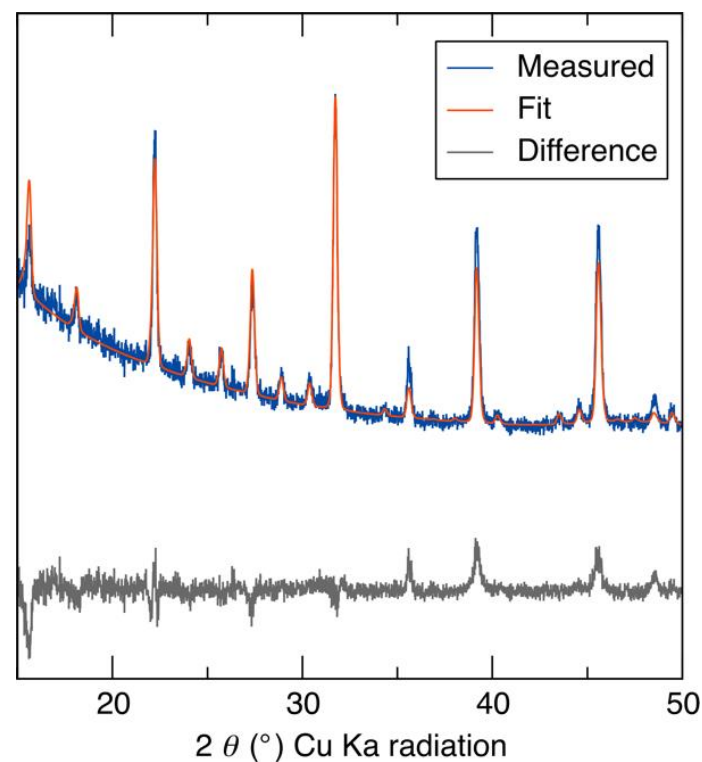

Figure S6. Rietveld refinement for compound with $x=0.31$ showing the PXRD data, the Rietveld fit produced by TOPAS, and the difference between them. 


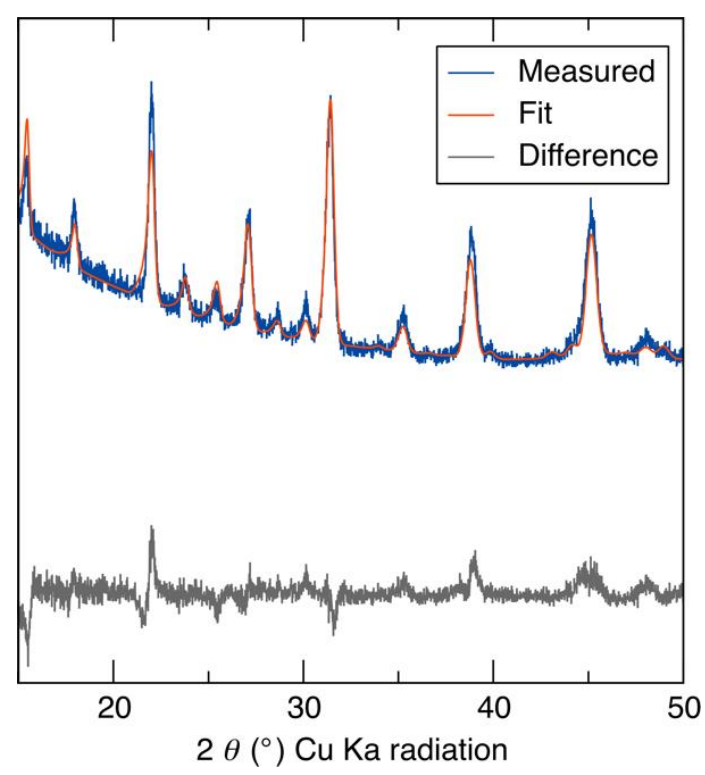

Figure S7. Rietveld refinement for compound with $x=0.36$ showing the PXRD data, the Rietveld fit produced by TOPAS, and the difference between them.

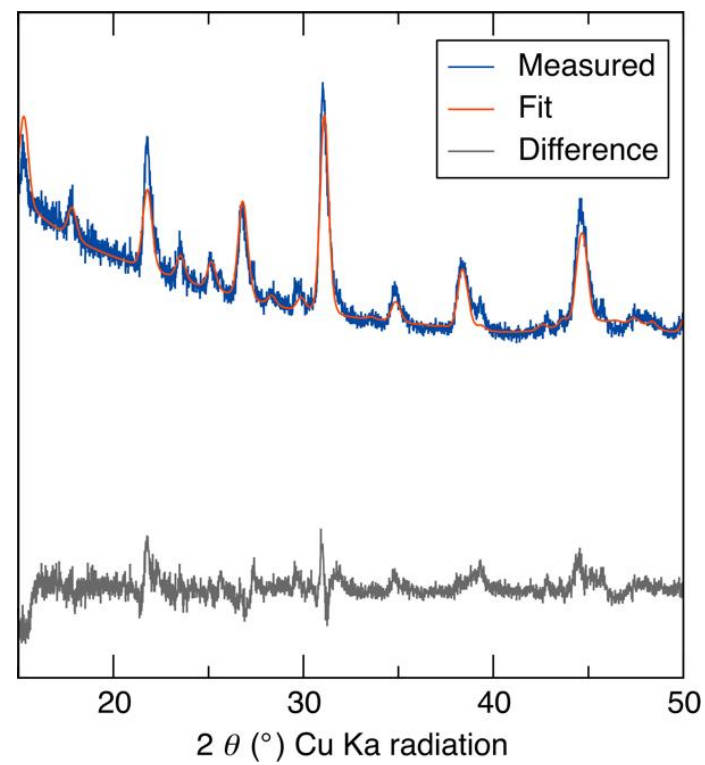

Figure S8. Rietveld refinement for compound with $x=0.52$ showing the PXRD data, the Rietveld fit produced by TOPAS, and the difference between them. 


\section{Section S3. Additional calculation data}

Table S7. PBE band gaps (in eV), valence band minima and conduction band maxima for compounds simulated in the $\mathrm{Pm}-31$ structure with lattice parameters from $\mathrm{Cs}_{3} \mathrm{Bi}_{2} \mathrm{Cl}_{8.16} \mathrm{I}_{0.84}$ single crystal diffraction. For indirect bandgaps, the smallest direct band gap is additionally listed.

\begin{tabular}{c|cccc|cccc}
\hline \multirow{2}{*}{ Composition } & \multicolumn{4}{|c|}{ w/o SOC } & \multicolumn{4}{c}{ w/SOC } \\
& DBG & IBG & VBM & CBM & DBG & IBG & VBM & CBM \\
\hline $\mathrm{Cs}_{3} \mathrm{Bi}_{2} \mathrm{Cl}_{9}$ & 2.966 & 2.966 & $\Gamma-M$ & $\Gamma$ & 2.184 & 2.074 & $\Gamma-M$ & $A$ \\
$\mathrm{Cs}_{3} \mathrm{Bi}_{2} \mathrm{Cl}_{6} \mathrm{I}_{3}$ & 2.405 & 2.404 & $K-\Gamma$ & $\Gamma$ & 1.487 & 1.413 & $\Gamma$ & $A$ \\
$\mathrm{Cs}_{3} \mathrm{Bi}_{2} \mathrm{Cl}_{3} \mathrm{I}_{6}$ & 1.874 & 1.841 & $K-\Gamma$ & $\Gamma$ & 1.57 & 1.554 & $K-\Gamma$ & $\Gamma$ \\
$\mathrm{Cs}_{3} \mathrm{Bi}_{2} \mathrm{I}_{9}$ & 1.657 & 1.552 & $K-\Gamma$ & $\Gamma$ & 1.331 & 1.24 & $K-\Gamma$ & $A$ \\
\hline
\end{tabular}

Table S8. PBE band gaps (in eV), valence band minima and conduction band maxima for $\mathrm{Cs}_{3} \mathrm{Bi}_{2} \mathrm{Cl}_{9}$ simulated in the Pnma structure with lattice parameters from $\mathrm{Cs}_{3} \mathrm{Bi}_{2} \mathrm{Cl}_{9}$ single crystal diffraction. For indirect bandgaps, the smallest direct band gap is additionally listed.

\begin{tabular}{c|cccc|cccc}
\hline \multirow{2}{*}{ Composition } & \multicolumn{4}{|c|}{ w/o SOC } & \multicolumn{4}{c}{ w/SOC } \\
& DBG & IBG & VBM & CBM & DBG & IBG & VBM & CBM \\
\hline $\mathrm{Cs}_{3} \mathrm{Bi}_{2} \mathrm{Cl}_{9}$ & 2.966 & 2.966 & $\Gamma-M$ & $\Gamma$ & 2.184 & 2.074 & $\Gamma-M$ & $A$ \\
$\mathrm{Cs}_{3} \mathrm{Bi}_{2} \mathrm{Cl}_{6} \mathrm{I}_{3}$ & 2.405 & 2.404 & $K-\Gamma$ & $\Gamma$ & 1.487 & 1.413 & $\Gamma$ & $A$ \\
$\mathrm{Cs}_{3} \mathrm{Bi}_{2} \mathrm{Cl}_{3} \mathrm{I}_{6}$ & 1.874 & 1.841 & $K-\Gamma$ & $\Gamma$ & 1.57 & 1.554 & $K-\Gamma$ & $\Gamma$ \\
$\mathrm{Cs}_{3} \mathrm{Bi}_{2} \mathrm{I}_{9}$ & 1.657 & 1.552 & $K-\Gamma$ & $\Gamma$ & 1.331 & 1.24 & $K-\Gamma$ & $A$ \\
\hline
\end{tabular}

Table S9. HSEo6 band gaps (in eV), valence band minima and conduction band maxima for the compounds simulated in $\mathrm{Pm}-31$ structures with lattice parameters from $\mathrm{Cs}_{3} \mathrm{Bi}_{2} \mathrm{Cl}_{8.16} \mathrm{I}_{0.84}$ single crystal diffraction. For indirect bandgaps, the smallest direct band gap is additionally listed.

\begin{tabular}{c|cccc}
\hline Composition & \multicolumn{4}{|c}{ w/SOC } \\
& DBG & IBG & VBM & CBM \\
\hline $\mathrm{Cs}_{3} \mathrm{Bi}_{2} \mathrm{Cl}_{9}$ & 2.938 & 2.827 & $G$ & $A$ \\
$\mathrm{Cs}_{3} \mathrm{Bi}_{2} \mathrm{I}_{9}$ & 1.764 & 1.677 & $K-G$ & $A$ \\
\hline
\end{tabular}

Table S1o. PBE band gaps (in eV), valence band minima and conduction band maxima for compounds simulated in hypothetical $P m-31 \mathrm{Cs}_{3} \mathrm{Bi}_{2} \mathrm{Cl}_{9-\mathrm{x}} \mathrm{I}_{\mathrm{x}}$ structures with linearly-interpolated lattice parameters for iodine incorporation between $0 \%(x=0)$ and $100 \%(x=9)$ based on powder Rietveld diffraction trendlines. For indirect bandgaps, the smallest direct band gap is additionally listed.

\begin{tabular}{c|c|cccc}
\hline o\% I / 100\% Cl & \multirow{2}{*}{ Composition } & DBG & IBG & VBM & CBM \\
\hline$a=7.68 \AA$ & $\mathrm{Cs}_{3} \mathrm{Bi}_{2} \mathrm{Cl}_{9}$ & 2.204 & 2.086 & $\Gamma-M$ & $A$ \\
$c=9.46 \AA$ & $\mathrm{Cs}_{3} \mathrm{Bi}_{2} \mathrm{Cl}_{6} \mathrm{I}_{3}$ & 1.473 & 1.395 & $\Gamma$ & $A$
\end{tabular}




\begin{tabular}{|c|c|c|c|c|c|}
\hline & $\begin{array}{c}\mathrm{Cs}_{3} \mathrm{Bi}_{2} \mathrm{Cl}_{3} \mathrm{I}_{6} \\
\mathrm{Cs}_{3} \mathrm{Bi}_{2} \mathrm{I}_{9} \\
\end{array}$ & $\begin{array}{l}1.530 \\
1.305 \\
\end{array}$ & $\begin{array}{l}1.507 \\
1.205 \\
\end{array}$ & $\begin{array}{l}K-\Gamma \\
K-\Gamma \\
\end{array}$ & $\begin{array}{l}\Gamma \\
\Gamma \\
\end{array}$ \\
\hline \multirow[t]{2}{*}{$20 \% \mathrm{I} / 80 \% \mathrm{Cl}$} & \multirow{2}{*}{ Composition } & \multicolumn{4}{|c|}{$\mathrm{w} / \mathrm{SOC}$} \\
\hline & & DBG & IBG & VBM & CBM \\
\hline \multirow{4}{*}{$\begin{aligned} a & =7.85 \AA \\
c & =9.61 \AA\end{aligned}$} & \multirow{4}{*}{$\begin{array}{c}\mathrm{Cs}_{3} \mathrm{Bi}_{2} \mathrm{Cl}_{9} \\
\mathrm{Cs}_{3} \mathrm{Bi}_{2} \mathrm{Cl}_{6} \mathrm{I}_{3} \\
\mathrm{Cs}_{3} \mathrm{Bi}_{2} \mathrm{Cl}_{3} \mathrm{I}_{6} \\
\mathrm{Cs}_{3} \mathrm{Bi}_{2} \mathrm{I}_{9}\end{array}$} & 2.155 & 2.053 & $\Gamma-M$ & $A$ \\
\hline & & 1.499 & 1.428 & $\Gamma$ & $A$ \\
\hline & & 1.620 & 1.609 & $K-\Gamma$ & $A$ \\
\hline & & 1.366 & 1.271 & $K-\Gamma$ & $A$ \\
\hline \multirow[t]{2}{*}{$40 \% \mathrm{I} / 60 \% \mathrm{Cl}$} & \multirow{2}{*}{ Composition } & \multicolumn{4}{|c|}{$\mathrm{w} / \mathrm{SOC}$} \\
\hline & & DBG & IBG & VBM & CBM \\
\hline \multirow{4}{*}{$\begin{array}{l}a=8.03 \AA \\
c=9.77 \AA\end{array}$} & \multirow{4}{*}{$\begin{array}{c}\mathrm{Cs}_{3} \mathrm{Bi}_{2} \mathrm{Cl}_{9} \\
\mathrm{Cs}_{3} \mathrm{Bi}_{2} \mathrm{Cl}_{6} \mathrm{I}_{3} \\
\mathrm{Cs}_{3} \mathrm{Bi}_{2} \mathrm{Cl}_{3} \mathrm{I}_{6} \\
\mathrm{Cs}_{3} \mathrm{Bi}_{2} \mathrm{I}_{9}\end{array}$} & 2.080 & 1.99 & $\Gamma$ & $A$ \\
\hline & & 1.501 & 1.435 & $\Gamma$ & $A$ \\
\hline & & 1.672 & 1.63 & $K-\Gamma$ & $A$ \\
\hline & & 1.406 & 1.31 & $K-\Gamma$ & $A$ \\
\hline \multirow[t]{2}{*}{$60 \% \mathrm{I} / 40 \% \mathrm{Cl}$} & \multirow{2}{*}{ Composition } & \multicolumn{4}{|c|}{$\mathrm{w} / \mathrm{SOC}$} \\
\hline & & DBG & IBG & VBM & $\mathrm{CBM}$ \\
\hline \multirow{4}{*}{$\begin{array}{l}a=8.20 \AA \\
c=9.92 \AA\end{array}$} & \multirow{4}{*}{$\begin{array}{c}\mathrm{Cs}_{3} \mathrm{Bi}_{2} \mathrm{Cl}_{9} \\
\mathrm{Cs}_{3} \mathrm{Bi}_{2} \mathrm{Cl}_{6} \mathrm{I}_{3} \\
\mathrm{Cs}_{3} \mathrm{Bi}_{2} \mathrm{Cl}_{3} \mathrm{I}_{6} \\
\mathrm{Cs}_{3} \mathrm{Bi}_{2} \mathrm{I}_{9}\end{array}$} & 1.979 & 1.899 & $\Gamma$ & $A$ \\
\hline & & 1.480 & 1.418 & $\Gamma$ & $A$ \\
\hline & & 1.691 & 1.621 & $\Gamma$ & $A$ \\
\hline & & 1.424 & 1.329 & $K-\Gamma$ & $A$ \\
\hline \multirow[t]{2}{*}{$80 \% \mathrm{I} / 20 \% \mathrm{Cl}$} & \multirow{2}{*}{ Composition } & \multicolumn{4}{|c|}{$\mathrm{w} / \mathrm{SOC}$} \\
\hline & & DBG & IBG & VBM & CBM \\
\hline \multirow{4}{*}{$\begin{array}{c}a=8.37 \AA \\
c=10.07 \AA\end{array}$} & \multirow{4}{*}{$\begin{array}{c}\mathrm{Cs}_{3} \mathrm{Bi}_{2} \mathrm{Cl}_{9} \\
\mathrm{Cs}_{3} \mathrm{Bi}_{2} \mathrm{Cl}_{6} \mathrm{I}_{3} \\
\mathrm{Cs}_{3} \mathrm{Bi}_{2} \mathrm{Cl}_{3} \mathrm{I}_{6} \\
\mathrm{Cs}_{3} \mathrm{Bi}_{2} \mathrm{I}_{9} \\
\end{array}$} & 1.828 & 1.793 & $\Gamma$ & $A$ \\
\hline & & 1.439 & 1.381 & $\Gamma$ & $A$ \\
\hline & & 1.680 & 1.587 & $\Gamma$ & $A$ \\
\hline & & 1.422 & 1.326 & $\Gamma-M$ & $A$ \\
\hline $100 \% \mathrm{I} / \mathrm{o} \% \mathrm{Cl}$ & & & & & \\
\hline & composition & DBG & IBG & VBM & $\mathrm{CBM}$ \\
\hline$a=8.56 \AA$ & $\mathrm{Cs}_{3} \mathrm{Bi}_{2} \mathrm{Cl}_{9}$ & 1.639 & $N / A$ & $A$ & $A$ \\
\hline$c=10.22 \AA$ & $\mathrm{Cs}_{3} \mathrm{Bi}_{2} \mathrm{Cl}_{6} \mathrm{I}_{3}$ & 1.336 & 1.332 & $\Gamma-M$ & $A$ \\
\hline & $\mathrm{Cs}_{3} \mathrm{Bi}_{2} \mathrm{Cl}_{3} \mathrm{I}_{6}$ & 1.643 & 1.535 & $\Gamma$ & $A$ \\
\hline & $\mathrm{Cs}_{3} \mathrm{Bi}_{2} \mathrm{I}_{9}$ & $1.35^{2}$ & 1.301 & $\Gamma-M$ & $A$ \\
\hline
\end{tabular}

